\title{
SOME GENERALIZATIONS OF FULL NORMALITY $\left.{ }^{(}\right)$
}

\author{
BY \\ M. J. MANSFIELD
}

This paper deals with two hierarchies of generalizations of full normality: $\mathfrak{m}$-full normality and almost- $\mathfrak{m}$-full normality, $\mathfrak{m}$ any cardinal number $\geqq 2$. The notions of 2 -full normality and $\boldsymbol{\aleph}_{0}$-full normality were introduced in 1955 by A. J. Goldman [8] in connection with some questions pertaining to Cech fundamental groups. The concept of almost-2-full normality was introduced in 1952 by $\mathrm{H}$. J. Cohen [2] in answering a question of Dieudonné $[4, \S 12$.III] concerning uniformities for normal spaces. The words "almost-2fully normal" are of the author's own invention; Cohen himself gave the concept no name. The idea of generalizing these concepts to arbitrary cardinal numbers was given to the author by $M$. Henriksen.

The first section of this paper contains a brief review of the terminology employed in the sequel. $\$ 2$ is devoted, for the most part, to establishing the relations existing between m-fully normal spaces and more well-known types of spaces (e.g. spaces $X$ for which all neighborhoods of the diagonal in $X \times X$ form a uniformity for $X$, collectionwise normal spaces, etc.). The results of this section are due largely to Cohen. $\S 3$ is devoted to proving that every linearly ordered space is $\boldsymbol{\aleph}_{0}$-fully normal. This is, perhaps, the most remarkable result of the paper. It is shown incidentally that, for any ordinal $\alpha$,

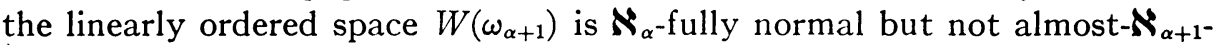
fully normal. $\S 4$ deals with subspaces and products of $\mathfrak{m}$-fully normal spaces. The main result, similar to [4, Théorème 2], is that every closed subspace of an $\mathfrak{m}$-fully normal (resp. almost-m-fully normal) space is itself $\mathfrak{m}$-fully normal (resp. almost-m-fully normal), and if each open subspace is $m$-fully normal (resp. almost-m-fully normal), then every subspace is $\mathfrak{m}$-fully normal (resp. almost-m-fully normal). Finally, in $\$ 5$, some additional properties of $\mathfrak{m}$-fully normal spaces are established. In particular, the questions of topological completeness and separation of arbitrary subsets by means of open

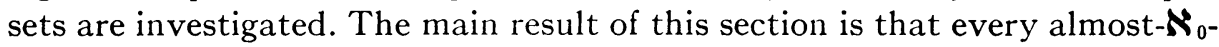
fully normal space is countably paracompact.

1. Terminology. Let $Q$ and $B$ be collections of subsets of a set $X$. The collection $Q$ is said to be a covering of $X$ if $\cup\{A: A \in Q\}=X$. The collection $B$ is said to be a refinement of $Q$ if $\bigcup\{B: B \in B\}=\cup\{A: A \in Q\}$ and each member of $B$ is a subset of some member of $Q$. Thus a refinement of a covering

Presented to the Society, April 19, 1957; received by the editors August 13, 1956.

(1) This paper is based on part of a thesis written by the author under the direction of Professor Melvin Henriksen and accepted by Purdue University in partial fulfillment of the requirements for the degree of Doctor of Philosophy. 
of $X$ is itself a covering of $X$. The star of a point $x \in X$ with respect to $B$, which is denoted by $\operatorname{St}(x, B)$, is the set $U\{B \in B: x \in B\}$. $B$ is a star refinement of $Q$ if $\{\mathrm{St}(x, B): x \in X\}$ is a refinement of $Q$. (Our usage of "star refinement," which follows [10, Exercise 5.U], differs from that of Tukey [16]. Tukey refers to our "star refinement" as a " $\Delta$-refinement," reserving the words "star refinement" to describe a slightly different concept.)

Following J. W. Tukey [16], a topological space $X$ is said to be fully normal if each open covering of $X$ admits an open star refinement.

Let $a$ be a collection of subsets of a topological space $X$. The collection $a$ is said to be locally finite if each $x \in X$ has a neighborhood meeting only finitely many members of $a$. Following J. Dieudonné [4], a topological space $X$ is said to be paracompact if (i) $X$ is a Hausdorff space and (ii) each open covering of $X$ admits a locally finite open refinement. Fully normal spaces and paracompact spaces are related by the following well-known theorem due to A. H. Stone. The reader is referred to [15] or [10, Exercise 5.U] for the proof.

Theorem 1.1 (Stone). A topological space $X$ is paracompact if and only if $X$ is a fully normal $T_{1}$-space.

\section{Definitions and fundamental relations.}

Definition 2.1. (a) Let $Q$ and $B$ be collections of subsets of a set $X$, and let $m$ be any cardinal number $\geqq 2$. $B$ is an $m$-star refinement of $Q$ if (i) $B$ is a refinement of $Q$ and (ii) for each $\mathcal{C} \subset \beta$ with $|\mathfrak{e}| \leqq \mathfrak{m}$ and $\cap\{C: C \in \mathfrak{e}\} \neq \varnothing$ there is an $A \in \mathbb{Q}$ such that $U\{C: C \in \mathbb{C}\} \subset A$.

(b) Let $m$ be any cardinal number $\geqq 2$. A topological space $X$ is said to be $\mathfrak{m}$-fully normal if each open covering of $X$ admits an open $m$-star refinement.

A fully normal space is evidently $m$-fully normal for every $\mathfrak{m} \geqq 2$. If $X$ is an $\mathfrak{m}$-fully normal space for some $\mathfrak{m}>2$, then $X$ is $\mathfrak{n}$-fully normal for each $\mathfrak{n}$, $2 \leqq \mathfrak{n}<\mathrm{m}$.

The next theorem is due to A. J. Goldman [8]. Since a proof is not included in [8] we supply one here.

THEOREM 2.2 (Goldman). If $X$ is a $k$-fully normal space for some finite cardinal $k \geqq 2$, then $X$ is $n$-fully normal for each finite cardinal $n=2,3, \cdots$.

Proof. It suffices to show that if $X$ is $k$-fully normal for some finite $k \geqq 2$, then $X$ is $2 k$-fully normal.

Let $R$ be an open covering of $X$. Since $X$ is a fortiori 2 -fully normal, $R$ admits an open 2 -star refinement $\delta$. Let $J$ be an open $k$-star refinement of $\delta$.

Suppose that $T_{1}, \cdots, T_{j} \in \mathcal{J}, j \leqq 2 k$, and $\cap\left\{T_{i}: i=1, \cdots, j\right\} \neq \varnothing$. Let $l$ be the largest integer $\leqq j / 2$. Then

$$
\cap\left\{T_{i}: i=1, \cdots, l\right\} \neq \varnothing, \quad \bigcap\left\{T_{i}: i=l+1, \cdots, j\right\} \neq \varnothing,
$$


and $|\{1, \cdots, l\}| \leqq k,|\{l+1, \cdots, j\}| \leqq k$. Since $J$ is a $k$-star refinement of $\delta$, there are sets $S_{1} \in \mathcal{S}$ and $S_{2} \in \mathcal{S}$ such that $U\left\{T_{i}: i=1, \cdots, l\right\} \subset S_{1}$ and $\cup\left\{T_{i}: i=l+1, \cdots, j\right\} \subset S_{2}$. Moreover $S_{1} \cap S_{2} \neq \varnothing$, so there is an $R \in \mathcal{Q}$ such that $R \supset S_{1} \cup S_{2} \supset \cup\left\{T_{i}: i=1, \cdots, j\right\}$. Hence $J$ is an open $2 k$-star refinement of $R$. Therefore $X$ is $2 k$-fully normal.

Definition 2.3. A topological space $X$ is said to be finitely-fully normal if for each open covering $R$ of $X$ there is an open covering $S$ (depending only on $R$ ) such that $\delta$ is an $n$-star refinement of $R$ for every finite cardinal $n \geqq 2$. Such a covering $S$ is said to be a finite-star refinement of $R$.

It is clear that a finitely-fully normal space is 2 -fully normal. The author can at present neither prove nor disprove the converse of this statement; he conjectures, however, that it is false.

- Each $\boldsymbol{\aleph}_{0}$-fully normal space is obviously finitely-fully normal. We shall employ an example due to C. H. Dowker [6] to show that the converse of this statement is false. Let $X$ be a space whose points are the real numbers. Let the open sets of $X$ be the empty set, the whole space $X$, and the subsets $G_{a}=\{x \in X: x<a\}$ for all real $a$. The space $X$ is finitely-fully normal-each open covering of $X$ is a finite-star refinement of itself-but not $\boldsymbol{\aleph}_{0}$-fully normal: the open covering $\left\{G_{i}: i=1,2, \cdots\right\}$, where $G_{i}=\{x \in X: x<i\}$, does not admit an open $\boldsymbol{\aleph}_{0}$-star refinement $\left({ }^{2}\right)$. It would be pleasant to give an example of a Hausdorff space which is finitely-fully normal but not $\boldsymbol{\aleph}_{0}$-fully normal; the author is, unfortunately, unable to do so. As we shall see later (cf. Corollary 5.3), a normal Hausdorff space which is not countably paracompact $\left({ }^{3}\right)$ would be a likely candidate for such an example. It is remarked in [8] that Morton Brown and Leonard Gillman have discovered an example of a Hausdorff space which is finitely-fully normal but not $\boldsymbol{\aleph}_{0}$-fully normal. Messrs. Brown and Gillman have been unable to supply the author with the details of this example.

Definition 2.4. (a) Let $Q$ and $Q$ be collections of subsets of a set $X$, and let $\mathfrak{m}$ be any cardinal number $\geqq 2$. $B$ is an almost- $m$-star refinement of $Q$ if (i) $B$ is a refinement of $Q$ and (ii) for each set $M$ with $|M| \leqq \mathfrak{m}$ and $M$ $\subset$ St $(x, \beta)$ for some $x$, there is an $A \in Q$ such that $M \subset A$.

(b) Let $m$ be any cardinal number $\geqq 2$. A topological space $X$ is said to be almost-m-fully normal if each open covering of $X$ admits an open almostm-star refinement.

It follows at once from the definitions that an $m$-fully normal space is almost-m-fully normal. Once again the author must confess his ignorance as to the validity of the converse, even for finite cardinals. Moreover, there is

(2) In fact, the covering $\left\{G_{i}: i=1,2, \cdots\right\}$ does not admit an open almost- $\boldsymbol{\aleph}_{0}$-star refinement (see Definition 2.4).

(3) Following Dowker [6], a topological (not necessarily Hausdorff) space $X$ is said to be countably paracompact if each countable open covering of $X$ admits a locally finite open refinement. 
no good reason at present to believe that the analog of Theorem 2.2 for almost- $k$-fully normal spaces is true. If $X$ is an almost-m-fully normal space for some cardinal $m>2$, then, obviously, $X$ is almost-n-fully normal for each $\mathfrak{n}, 2 \leqq \mathfrak{n}<\mathfrak{m}$.

Proposition 2.5. If $X$ is a topological space with $|X| \leqq \mathfrak{m}$, and if $X$ is almost-m-fully normal, then $X$ is fully normal. Consequently, if $X$ is a Hausdorff space of power $\leqq \mathfrak{m}$ which is almost-m-fully normal, then $X$ is paracompact.

Proof. The assertions follow easily from Definition 2.4 and Theorem 1.1.

The next theorem and its corollaries are due to $\mathrm{H}$. J. Cohen [2, Théorème 2 ]. Since the proofs are not given in Cohen's original paper, they are supplied here.

Theorem 2.6 (COHEN). Let $X$ be a topological space and let $\Delta$ denote the diagonal in $X \times X$. In order that for each neighborhood $U$ of $\Delta$ there exist an open symmetric neighborhood $V$ of $\Delta$ such that $V \circ V \subset U$, it is necessary and sufficient that $X$ be almost-2-fully normal $\left({ }^{4}\right)$.

Proof. Suppose that $X$ is almost-2-fully normal, and let $U$ be a neighborhood of $\Delta$ in $X \times X$. We must find an open symmetric neighborhood $V$ of $\Delta$ such that $V \circ V \subset U$. Since $U$ is a neighborhood of $\Delta$, for each $x \in X$ there is an open set $W_{x}$ containing $x$ such that $W_{x} \times W_{x} \subset U$. The family $w$ $=\left\{W_{x}: x \in X\right\}$ is an open covering of $X$. Let $\delta$ be an open almost-2-star refinement of $\mathscr{W}$, and let $V=\bigcup\{S \times S: S \in \mathcal{S}\}$. Obviously $V$ is an open symmetric neighborhood of $\Delta$.

If $(u, v) \in V \circ V$, then there is a $w \in X$ such that $(u, w) \in V$ and $(w, v) \in V$. By definition of $V$ this means that there is an $S_{1} \in \mathcal{S}$ such that $u \in S_{1}$ and $w \in S_{1}$, and there is an $S_{2} \in \mathcal{S}$ such that $w \in S_{2}$ and $v \in S_{2}$. Hence $\{u, v\}$ $\subset$ St $(w, \delta)$. Since $S$ is an almost-2-star refinement of $w$, there is an $x \in X$ such that $\{u, v\} \subset W_{x}$. Therefore $(u, v) \in W_{x} \times W_{x} \subset U$. Hence $V \circ V \subset U$.

Conversely, suppose that for each neighborhood $U$ of $\Delta$ there is an open symmetric neighborhood $V$ of $\Delta$ such that $V \circ V \subset U$. Let $R$ be an open covering of $X$. We must show that $R$ admits an open almost-2-star refinement. Now $U=U\{R \times R: R \in R\}$ is a neighborhood of $\Delta$ in $X \times X$. Choose an open symmetric neighborhood $V$ of $\Delta$ such that $V \circ V \circ V \circ V \subset U$. Then, for each $x \in X, V[x]$ is an open set containing $x$. Moreover, for each $x \in X$ there is an $R_{x} \in \mathbb{R}$ such that $x \in R_{x}$. If $W_{x}=V[x] \cap R_{x}$, then $\mathscr{W}=\left\{W_{x}: x \in X\right\}$ is an open refinement of $R$.

Suppose that $\{u, v\} \subset \mathrm{St}(w, w)$ for some $w \in X$. Then $u, w \in V\left[x_{1}\right]$ and $v, w \in V\left[x_{2}\right]$ for some $x_{1}, x_{2} \in X$. Since $V$ is symmetric, we then have $\left(u, x_{1}\right)$, $\left(x_{1}, w\right),\left(w, x_{2}\right),\left(x_{2}, v\right) \in V$. Hence $(u, v) \in V \circ V \circ V \circ V \subset U$. From the definition of $U$ it follows that there is an $R_{u, v} \in R$ such that $(u, v) \in R_{u, v} \times R_{u, v}$, i.e., $\{u, v\} \subset R_{u, v}$. Therefore $W$ is an open almost-2-star refinement of $R$. It follows that $X$ is almost-2-fully normal.

(4) Our notation and terminology relevant to uniform spaces follow [10, Chap. 6]. 
Corollary 2.7 (Cohen). Let $(X, J)$ be a topological space. The family $\mathcal{U}_{\Delta}(\mathfrak{J})$ of all J-neighborhoods of the diagonal $\Delta$ in $X \times X$ is a uniformity for $X$ if and only if $(X, J)$ is almost-2-fully normal.

Corollary 2.8 (Cohen). If $(X, J)$ is an almost-2-fully normal Hausdorff space, then $\mathfrak{U}_{\Delta}(\mathfrak{J})$ is a uniformity for $X$ whose topology is $\mathfrak{J}$.

Proof. Since $(X, \mathfrak{J})$ is almost-2-fully normal, it follows from Corollary 2.7 that $\mathfrak{U}_{\Delta}(\mathfrak{J})$ is a uniformity for $X$. If $N_{x}$ is a $\mathfrak{J}$-open neighborhood of some $x \in X$, consider the open covering $\left\{X-\{x\}, N_{x}\right\}$ of $X\left(^{5}\right)$. Let $V=\left(N_{x} \times N_{x}\right)$ $\mathrm{U}((X-\{x\}) \times(X-\{x\}))$. Clearly $V \in \mathfrak{u}_{\Delta}(J)$, and $V[x] \subset N_{x}$. Hence $N_{x}$ is a $\mathcal{U}_{\Delta}(J)$-neighborhood of $x$.

If $U \in \mathfrak{u}_{\Delta}(\mathfrak{J})$, consider $U[x]$ for any fixed $x \in X$. There is a J-open set $N_{x}$ containing $x$ such that $N_{x} \times N_{x} \subset U$. If $y \in N_{x}$, then $(x, y) \in N_{x} \times N_{x} \subset U$, i.e., $y \in U[x]$. Hence $N_{x} \subset U[x]$, and therefore $U[x]$ is a J-neighborhood of $x$. Thus the uniform topology is $J$.

A collection $Q$ of subsets of a topological space $X$ is said to be discrete if each $x \in X$ has a neighborhood meeting at most one member of $a$. Following R. H. Bing [1], a topological space $X$ is said to be collectionwise normal if for each discrete collection $\left\{A_{\beta}: \beta \in B\right\}$ of subsets of $X$ there is a collection $\left\{G_{\beta}: \beta \in B\right\}$ of pairwise disjoint open sets such that $A_{B} \subset G_{\beta}$ for each $\beta \in B$. A collectionwise normal space is normal, since two disjoint closed sets constitute a discrete collection.

The next theorem is also due to Cohen [2, Théorème 1 ]. The reader is referred to Cohen's original paper or our Theorem 5.2 for the proof. It is comforting to observe that an almost-m-fully normal space is, indeed, normal.

Theorem 2.9 (COHEN). If $X$ is an almost-2-fully normal space, then $X$ is collectionwise normal.

The converse of Theorem 2.9 is false: In [2] Cohen gives an example, due to R. H. Bing, of a collectionwise normal Hausdorff space which is not almost-2-fully normal.

3. Linearly ordered spaces. In this section we show that every linearly ordered space is $\boldsymbol{\aleph}_{0}$-fully normal, and that for any ordinal $\alpha$, the linearly

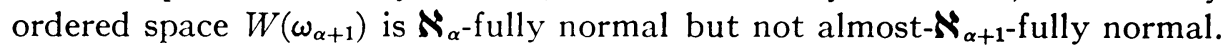

We recall briefly some facts concerning linearly ordered sets; the reader is referred to Hausdorff [9] for a more detailed discussion. The set of all ordinals less than a given ordinal $\phi$ is denoted by $W(\phi)$. The least ordinal $\phi$ such that $W(\phi)$ has power $\boldsymbol{\aleph}_{\alpha}$ is denoted by $\omega_{\alpha}\left(\omega_{0}=\omega\right)$ and is called the initial ordinal of power $\boldsymbol{\aleph}_{\alpha}$.

Let $Y$ be a subset of a linearly ordered set $X$. We say that $X$ is cofinal (resp. coinitial) with $Y$ if for each $x \in X$ there is a $y \in Y$ such that $y \geqq x$ (resp.

(5) This is the only place in the proof where the hypothesis that $X$ be a Hausdorff space is needed. 
$y \leqq x)$. An initial ordinal $\omega_{\alpha}$ is said to be regular if $W\left(\omega_{\alpha}\right)$ is cofinal with no subset of type $<\omega_{\alpha}$. The ordinal $\omega=\omega_{0}$ is regular, and so is every initial ordinal of the form $\omega_{\beta+1}$.

Let $X$ be a linearly ordered set. A Dedekind cut $(A \mid B)$ of $X$ such that $A$ has no last element and $B$ has no first element will be called, following Gillman and Henriksen [7], an interior gap of $X$. Such a gap may be regarded as a "virtual" element $u$ such that $a<u<b$ for all $a \in A$ and $b \in B$. If $X$ has no first (resp. last) element, we shall introduce a virtual element $u$ such that $u<x$ (resp. $u>x$ ) for all $x \in X$, and refer to $u$ as a left (resp. right) end-gap of $X$. The linearly ordered set consisting of all elements and all gaps (end-gaps as well as interior gaps) of $X$ will be denoted by $X^{+}$.

If $\mu$ denotes the order type of a set $X$ linearly ordered by $<$, then $\mu^{*}$ is used to denote the order type of $X$ when ordered by $>$. A linearly ordered set $X$ is said to be cofinal with $\omega_{\alpha}$ (resp. coinitial with $\omega_{\alpha}^{*}$ ) if $X$ is cofinal (resp. coinitial) with a subset of type $\omega_{\alpha}$ (resp. $\omega_{\alpha}^{*}$ ). If $X$ is an infinite ordered set having no last (resp. first) element, then there is a unique regular initial ordinal $\omega_{\alpha}$ such that $X$ is cofinal (resp. coinitial) with $\omega_{\alpha}$ (resp. $\omega_{\alpha}^{*}$ ).

Let $X$ be a linearly ordered set. By the linearly ordered space $X$ we shall mean the set $X$ provided with the topology which has as a subbase the family of all sets of the form $\{x \in X: x<a\}$ or $\{x \in X: x>a\}, a \in X$. Every linearly ordered space is a normal Hausdorff space.

The symbols ( ), [ ], etc. will be used in the usual way to denote intervals (open, closed, etc.) of $X$. The indicated boundaries of the interval will be in $X^{+}$, but they need not be in $X$ itself. The word proper will be used to describe intervals whose boundaries lie in $X$.

Let $X$ be a linearly ordered space having no interior gaps. If $X$ is not cofinal (resp. coinitial) with $\omega$ (resp. $\omega^{*}$ ), then every strictly increasing $\omega$ - (resp. decreasing $\omega^{*}$-) sequence in $X$ converges in $X$. A linearly ordered space having no gaps is compact (cf. e.g. [7, Lemma 9.2]).

The following lemma is a generalization of a result due to Dieudonné $[5$, no. 3].

LeMma 3.1. Let $X$ be a linearly ordered space with the property that every strictly increasing $\omega$ - (resp. decreasing $\omega^{*}$-) sequence in $X$ converges in $X$. Then for each open covering $\mathbb{R}$ of $X$ there is a point $x^{*}(\Re) \in X$ such that $\{x \in X$ : $\left.x>x^{*}(\Omega)\right\} \subset \operatorname{St}\left(x^{*}(R), \Omega\right)$ (resp. such that

$$
\left\{x \in X: x<x^{*}(\Re)\right\} \subset \operatorname{St}\left(x^{*}(\mathcal{R}),(R)\right) .
$$

Proof. Suppose that the conclusion of the lemma is false. Then there is an open covering $\Omega_{0}$ of $X$ such that for each $x \in X$ there is a $y \in X, y>x$ (resp. $y<x$ ), such that $y \in \operatorname{St}\left(x, R_{0}\right)$. Choose $x_{1} \in X$. Then there is an $x_{2} \in X$, $x_{2}>x_{1}$ (resp. $\left.x_{2}<x_{1}\right)$, such that $x_{2} \in \operatorname{St}\left(x_{1}, \Omega_{0}\right)$. Proceeding inductively, there is an $x_{n+1} \in X, x_{n+1}>x_{n}$ (resp. $\left.x_{n+1}<x_{n}\right)$, such that $x_{n+1} \notin \operatorname{St}\left(x_{n}, R_{0}\right)$. By hypothesis, $\lim _{k<\omega} x_{k}$ exists, say $z_{0}=\lim _{k<\omega} x_{k}$. Since $\Omega_{0}$ is a covering of $X$, 
there is an $R_{0} \in R_{0}$ such that $z_{0} \in R_{0}$. Since $R_{0}$ is open and $z_{0}$ is not the first (resp. last) element of $X$, there is an $a_{0} \in X, a_{0}<z_{0}$ (resp. $a_{0}>z_{0}$ ), such that $\left(a_{0}, z_{0}\right] \subset R_{0}$ (resp. $\left.\left[z_{0}, a_{0}\right) \subset R_{0}\right)$. By definition of $z_{0}$, there is a $k_{0}<\omega$ such that $k \geqq k_{0}$ implies that $x_{k} \in\left(a_{0}, z_{0}\right]$ (resp. $x_{k} \in\left[z_{0}, a_{0}\right)$ ). In particular, $x_{k_{0}}, x_{k_{0}+1}$ $\in\left(a_{0}, z_{0}\right] \subset R_{0}$ (resp. $\left.x_{k_{0}}, x_{k_{0}+1} \in\left[z_{0}, a_{0}\right) \subset R_{0}\right)$. Therefore $x_{k_{0}+1} \in \operatorname{St}\left(x_{k_{0}}, R_{0}\right)$. This contradicts the choice of $x_{k_{0}+1}$. The lemma follows.

Lemma 3.2. Let $K=(a, b)$ be an interval of a linearly ordered space $X$, and suppose that there is an ordinal $\alpha$ such that $K$ is cofinal (resp. coinitial) with no subset of power $\leqq \boldsymbol{N}_{\alpha}$. If $\mathfrak{g}$ is a covering of $K$ with the property that $I \in \mathcal{g}$ implies that there is an $x_{I} \in X, a<x_{I}<b$, such that $I=\left(a, x_{I}\right)$ (resp. $\left.I=\left(x_{I}, b\right)\right)$, then $g$ is an $\mathbf{\aleph}_{\alpha}$-star refinement of itself.

Proof. Let $g$ be a covering of $K$ such that each member $I$ of $g$ is of the form $\left(a, x_{I}\right)$ (resp. $\left(x_{I}, b\right)$ ) for some $x_{I} \in X, a<x_{I}<b$. We must show that $g$ is an $\boldsymbol{\aleph}_{\alpha}$-star refinement of itself.

Suppose that $\mathfrak{d} \subset \mathcal{g}$ and $|\mathfrak{g}| \leqq \boldsymbol{\aleph}_{\alpha}$. By hypothesis there is an $x(\mathfrak{g}) \in K$ such that $x(\mathcal{J})>x_{I}$ (resp. $\left.x(\mathcal{J})<x_{I}\right)$ for all $I \in \mathcal{J}$. Since $\mathscr{g}$ is a covering of $K$, there is an $I_{0}=\left(a, x_{I_{0}}\right) \in \mathfrak{g} \quad$ (resp. $\left.I_{0}=\left(x_{I_{0}}, b\right) \in \mathfrak{g}\right)$ such that $x(\mathfrak{g}) \in I_{0}$. Hence $\cup\{I: I \in \mathcal{J}\} \subset I_{0}$. Therefore $\mathfrak{g}$ is an $\boldsymbol{\aleph}_{\alpha}$-star refinement of itself.

Theorem 3.3. For any ordinal $\alpha$, the linearly ordered space $W\left(\omega_{\alpha+1}\right)$ is $\boldsymbol{\aleph}_{\alpha}$-fully normal but not almost $\boldsymbol{\aleph}_{\alpha+1}$-fully normal.

Proof. Let $R$ be an open covering of $W\left(\omega_{\alpha+1}\right)$. We must show that $R$ admits an open $\boldsymbol{\aleph}_{\alpha}$-star refinement $\delta$.

Let $I_{0}=\{0\}=[0,1)$. For $x \in W\left(\omega_{\alpha+1}\right)-\{0\}$, choose $R_{x} \in \mathbb{R}$ such that $x \in R_{x}$. Since $R_{x}$ is an open set, there are ordinals $\xi_{x}, \eta_{x}, \xi_{x}<\eta_{x}$, such that $x \in\left(\xi_{x}, \eta_{x}\right) \subset R_{x}$. Let $I_{x}=\left(\xi_{x}, \eta_{x}\right)$ and $\mathfrak{g}=\left\{I_{x}: x \in W\left(\omega_{\alpha+1}\right)\right\}$. Evidently $\mathfrak{g}$ is an open refinement of $R$.

Now $W\left(\omega_{\alpha+1}\right)$ is a well-ordered set. Therefore $W\left(\omega_{\alpha+1}\right)$ has no interior gaps. Since $\omega_{\alpha+1}$ is regular, it follows that $W\left(\omega_{\alpha+1}\right)$ is not cofinal with $\omega$. Therefore every strictly increasing $\omega$-sequence in $W\left(\omega_{\alpha+1}\right)$ converges. Hence, by Lemma 3.1, there is an $x^{*}(\mathfrak{g}) \in W\left(\omega_{\alpha+1}\right)$ such that $\left\{x \in W\left(\omega_{\alpha+1}\right): x>x^{*}(\mathfrak{g})\right\}$ $C$ St $\left(x^{*}(\mathfrak{g}), \mathfrak{g}\right)$. Let $\mathfrak{g}_{1}=\left\{I \cap\left[0, x^{*}(\mathfrak{g})\right]: I \in \mathfrak{g}\right\}$. Then $\mathfrak{g}_{1}$ is an open (in $\left.\left[0, x^{*}(\mathfrak{g})\right]\right)$ covering of $\left[0, x^{*}(\mathfrak{g})\right]$. Since $\left[0, x^{*}(\mathfrak{g})\right]$ has no gaps, it follows that $\left[0, x^{*}(\mathfrak{g})\right]$ is compact, hence paracompact, hence fully normal. Therefore $g_{1}$ admits an open (in $\left[0, x^{*}(g)\right]$ ) star refinement $\delta_{1}$. Since $\left[0, x^{*}(g)\right]$ $=\left[0, x^{*}(\mathcal{g})+1\right)$ is open in $W\left(\omega_{\alpha+1}\right)$, the sets $S \in \mathcal{S}_{1}$ are open in $W\left(\omega_{\alpha+1}\right)$.

Now, by definition of $x^{*}(\mathfrak{g})$, for each $x>x^{*}(\mathfrak{g})$ there is an interval $I \in \mathfrak{g}$ such that $x^{*}(\mathfrak{g}) \in I$ and $x \in I$. Therefore the interval $J_{x}=\left(x^{*}(\mathfrak{g}), x\right)$ is contained in $I$. Let $S_{2}=\left\{J_{x}: x>x^{*}(g)\right\}$. Since $\omega_{\alpha+1}$ is regular, it follows from Lemma 3.2 that $\delta_{2}$ is an $\boldsymbol{\aleph}_{\alpha}$-star refinement of itself.

Let $S=S_{1} \cup \mathcal{S}_{2}$. It follow's that $\mathcal{S}$ is an open $\boldsymbol{\aleph}_{\alpha}$-star refinement of $g$ and hence of $\mathbb{R}$. Therefore $W\left(\boldsymbol{\omega}_{\alpha+1}\right)$ is $\boldsymbol{\aleph}_{\alpha}$-fully normal. 
We shall now show that $W\left(\omega_{\alpha+1}\right)$ is not almost- $\boldsymbol{\aleph}_{\alpha+1}$-fully normal. Let $\mathcal{R}$ be the open covering $\left\{[0, \alpha): \alpha \in W\left(\omega_{\alpha+1}\right)-\{0\}\right\}$, and let $\delta$ be any open refinement of $R$. It follows from Lemma 3.1 that there is an $x^{*}(\delta) \in W\left(\omega_{\alpha+1}\right)$ such that $\left\{x \in W\left(\omega_{\alpha+1}\right): x>x^{*}(\delta)\right\} \subset \operatorname{St}\left(x^{*}(\delta), \delta\right)$. Now the set $\left\{x \in W\left(\omega_{\alpha+1}\right)\right.$ : $\left.x>x^{*}(s)\right\}$ has power $\boldsymbol{\aleph}_{\alpha+1}$. But each $R \in R$ has power $\left\langle\boldsymbol{\aleph}_{\alpha+1}\right.$. Thus there is no $R \in R$ such that $\left\{x \in W\left(\omega_{\alpha+1}\right): x>x^{*}(\mathcal{S})\right\} \subset R$. Hence $S$ is not an almost-

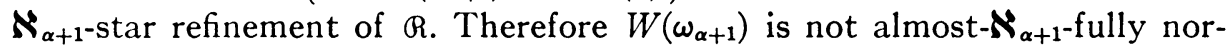
mal.

Let $J$ be an interval of a linearly ordered space $X$. Following Gillman and Henriksen [7], a gap $u$ of $J$ is said to be covered in $J$ by an interval $K=(x, y)$ of $X$ if either $x<u<y$, or one of $x, y$ is an end-gap of $J$ and coincides with $u$. The gap $u$ is covered in $J$ by an open subset $R$ of $X$ if there is an interval $K$ of $X$, contained in $R$, such that $u$ is covered in $J$ by $K$. The gap $u$ is covered in $J$ by a family $R$ of open subsets of $X$ if it is covered in $J$ by some member of $R$.

\section{THEOREM 3.4. Each linearly ordered space $X$ is $\boldsymbol{\aleph}_{0}$-fully normal.}

Proof. If $X$ has at most one point, then the assertion is trivial. Therefore we assume that $X$ contains at least two distinct points.

Let $R$ be an open covering of $X$. We must show that $R$ admits an open $\aleph_{0}$-star refinement $\mathcal{J}$.

Now $R$ admits an open refinement $g$ such that each member of $g$ is a proper interval of $X$; i.e., each member of $g$ is of the form $(a, b)$ for some $a, b \in X$ such that $a<b$, with the obvious modifications to take care of the first element and/or last element of $X$, in case such elements exist.

We now employ a construction due to Gillman and Henriksen [7, Proof of Theorem 9.5]. Denote by $F^{+}$the set of all gaps of $X$ which are not covered in $X$ by $g$. Note that since the boundary points of the members of $g$ are points in $X$, a gap $u$ of $X$ is covered in $X$ by $\mathscr{g}$ if and only if there is an $I=(a, b) \in \mathfrak{g}$ such that $a<u<b$.

We shall show that $F^{+}$is closed in $X^{+}$. If $x \in X^{+}-F^{+}$, then either $x$ is a point of $X$ or $x$ is a covered gap. In either case, there is an $I_{x}=(a, b) \in \mathfrak{g}$ such that $a<x<b$. If $y \in X^{+}$and $a<y<b$, then either $y \in X$ or $y$ is a covered gap. Hence $\left\{y \in X^{+}: a<y<b\right\} \cap F^{+}=\varnothing$. Therefore $F^{+}$is closed in $X^{+}$.

Since $X^{+}-F^{+}$is open in $X^{+}$, there is a family $\left\{K_{\alpha}^{+}: \alpha \in A\right\}$ of pairwise disjoint open intervals of $X^{+}$such that $X^{+}-F^{+}=\cup\left\{K_{\alpha}^{+}: \alpha \in A\right\}$. Now each $K_{\alpha}^{+}$, in the relative topology of $X^{+}$, is a linearly ordered space having no interior gaps. Assume for the moment that such a space is $\boldsymbol{\aleph}_{0}$-fully normal, and notice how the theorem follows.

For each $I=(a, b) \in \mathfrak{g}$, let $I^{+}=\left\{x \in X^{+}: a<x<b\right\}$. Then, since each point of $X^{+}-F^{+}$is either a point of $X$ or a covered gap, $g^{+}=\left\{I^{+}: I \in g\right\}$ is an open (in $X^{+}$) covering of $X^{+}-F^{+}$. For each $\alpha \in A$, let $g_{\alpha}^{+}=\left\{I^{+} \cap K_{\alpha}^{+}: I \in g\right\}$. Obviously $\mathfrak{I}_{\alpha}^{+}$is an open covering of $K_{a}^{+}$. Hence, by the assumption of the preced- 
ing paragraph, each $\mathfrak{g}_{\alpha}^{+}$admits an open (in $K_{\alpha}^{+}$and hence in $X^{+}$) $\boldsymbol{\aleph}_{0}$-star refinement $g_{\alpha}^{+}$. Since the $K_{\alpha}^{+}$are disjoint, $g^{+}=U\left\{g_{\alpha}^{+}: \alpha \in A\right\}$ is an open $\boldsymbol{\aleph}_{0}$-star refinement of $\mathfrak{g}^{+}$. Therefore $\mathcal{J}=\left\{J^{+} \cap X: J^{+} \in \mathcal{J}^{+}\right\}$is an open (in $X$ ) $\boldsymbol{\aleph}_{0}$-star refinement of $g$ and hence of $R$.

Thus, in order to complete the proof of Theorem 3.4, it suffices to prove the following lemma.

Lemma 3.5. If $X$ is a linearly ordered space having no interior gaps, then $X$ is $\boldsymbol{\aleph}_{0}$-fully normal.

Proof. If $X$ has at most one point, then the assertion is trivial. Therefore we assume that $X$ contains at least two distinct points. We shall denote the first element, or left end-gap, as the case may be, of $X$ by $u$, and the last element, or right end-gap, by $v$.

Let $R$ be an open covering of $X$. We must show that $R$ admits an open $\aleph_{0}$-star refinement. First of all, we observe that $R$ admits an open refinement $g$ such that each member of $\mathscr{g}$ is an interval of $X$. We now proceed by cases.

Case I. Suppose that both $u$ and $v$ are gaps, and that $X$ is neither cofinal with $\omega$ nor coinitial with $\omega^{*}$. Then every strictly increasing $\omega$-sequence and every strictly decreasing $\omega^{*}$-sequence in $X$ converge in $X$. Therefore, by Lemma 3.1, there are points $r$ and $l$ in $X$ such that $\{x: x>r\} \subset \operatorname{St}(r, g)$ and $\{x: x<l\} \subset \operatorname{St}(l, g)$. There is no loss of generality in assuming that $l<r$, because $X$ has no last element: If $r^{\prime}>r$ and $x>r^{\prime}$, then, by definition of $r$, there is an interval $I \in \mathfrak{g}$ such that $x \in I$ and $r \in I$. Since $r<r^{\prime}<x$, it follows that $r^{\prime} \in I$, and so $x \in \operatorname{St}\left(r^{\prime}, g\right)$. Hence $\left\{x: x>r^{\prime}\right\} \subset$ St $\left(r^{\prime}, g\right)$. Similarly, if $l^{\prime}<l$, then $\left\{x: x<l^{\prime}\right\} \subset \operatorname{St}\left(l^{\prime}, \mathfrak{g}\right)$.

Choose $l^{*}, r^{*}, p, q, s, t \in X$ such that $r<s<r^{*}<t$ and $l>q>l^{*}>p$. This can be done because $X$ has neither a first nor a last element. As shown above, $r^{*}$ and $l^{*}$ have the properties that $\left\{x \in X: x>r^{*}\right\} \subset$ St $\left(r^{*}, g\right)$ and $\{x \in X$ : $\left.x<l^{*}\right\} \subset$ St $\left(l^{*}, g\right)$. For each $x>r^{*}$ (resp. $\left.x<l^{*}\right)$, then, there is an interval $I_{x} \in \mathscr{g}$ such that $r^{*}, x$ (resp. $\left.l^{*}, x\right) \in I_{x}$. Let $J_{x}=\left(r^{*}, x\right)$ (resp. $\left.\left(x, l^{*}\right)\right)$. Then $J_{x} \subset I_{x}$. Let $g_{r^{*}}=\left\{J_{x}: x>r^{*}\right\}$ and $J_{l^{*}}=\left\{J_{x}: x<l^{*}\right\}$. Evidently $g_{r^{*}}$ is an open (in $X$ ) covering of $\left(r^{*}, v\right)$ and $J l^{*}$ is an open (in $X$ ) covering of $\left(u, l^{*}\right)$.

Since $X$ has no last (resp. first) element and is not cofinal (resp. coinitial) with $\omega$ (resp. $\omega^{*}$ ), it follows that $X$ is cofinal (resp. coinitial) with no subset of power $\leqq \boldsymbol{\aleph}_{0}$. Hence, by Lemma $3.2, g_{r^{*}}$ and $\mathcal{d} l^{*}$ are $\boldsymbol{\aleph}_{0}$-star refinements of themselves.

Let $\mathfrak{g}_{0}=(\{[p, q)\}) \cup\left(\left\{I \cap\left(l^{*}, r^{*}\right): I \in \mathfrak{g}\right\}\right) \cup(\{(s, t]\})$. Since $r<s<t$ and $p<q<l$, it follows from the definition of $r$ and $l$ that $(s, t] \subset I_{1}$ and $[p, q) \subset I_{2}$ for some intervals $I_{1}, I_{2} \in \mathfrak{g}$. Now $\mathfrak{g}_{0}$ is an open (in $[p, t]$ ) covering of the linearly ordered space $[p, t]$. Since $[p, t]$ has no gaps, it follows that $[p, t]$ is compact, hence paracompact, hence fully normal. Therefore $\mathscr{g}_{0}$ admits an open (in $[p, t]$ ) star refinement $\mathscr{K}_{0}$ whose members are intervals of $[p, t]$.

Let $\mathcal{J}_{0}=\left\{K-(\{p\} \cup\{t\}): K \in \mathcal{K}_{0}\right\}$. Then $\mathcal{J}_{0}$ is a covering of $(p, t)$ such 
that $\mathrm{St}\left(x, g_{0}\right)$ is contained in some member of $g_{0}$ for each $x \in(p, t)$. Moreover, each nonempty $J \in \mathcal{J}_{0}$ is a proper open interval of $X:$ If, for example, $t \in K \in \mathfrak{K}_{0}$, then $K=(a, t]$ for some $a, p<a<t$. Consequently $K-(\{p\} \cup\{t\})$ $=(a, t)$.

Let $g=J_{\imath} \cup \mathcal{J}_{0} \cup \mathcal{J}^{*}$. Evidently $\mathcal{g}$ is an open refinement of $g$. Suppose that $\left\{\left(a_{k}, b_{k}\right): k=1,2, \cdots\right\}$ is a countable collection of members of $\mathcal{J}$ such that $\bigcap\left\{\left(a_{k}, b_{k}\right): k=1,2, \cdots\right\} \neq \varnothing$. We must find an $I \in g$ such that $\cup\left\{\left(a_{k}, b_{k}\right)\right.$ : $k=1,2, \cdots\} \subset I$. If $\left(a_{k}, b_{k}\right) \in \mathcal{J}^{*}, k=1,2, \cdots$; or if $\left(a_{k}, b_{k}\right) \in \mathcal{J}_{0}, k=$ $=1,2, \cdots ;$ or if $\left(a_{k}, b_{k}\right) \in \partial_{r^{*}}, k=1,2, \cdots ;$ then, by construction of $\mathrm{d}^{*}$, $\mathfrak{I}_{0}$, and $\mathcal{I}_{r^{*}}$, there is an $I \in \mathcal{I}$ such that $\cup\left\{\left(a_{k}, b_{k}\right): k=1,2, \cdots\right\} \subset I$. If

$$
\begin{aligned}
\left\{\left(a_{k}, b_{k}\right): k=1,2, \cdots\right\}= & \left(\left\{\left(a_{m_{\nu}}, b_{m_{\nu}}\right): \nu=1,2, \cdots\right\}\right) \\
& \cup\left(\left\{\left(a_{n_{\mu}}, b_{n_{\mu}}\right): \mu=1,2, \cdots\right\}\right)
\end{aligned}
$$

where $\left(a_{m_{\nu}}, b_{m_{\nu}}\right) \in \mathcal{I}_{0}, \nu=1,2, \cdots,\left(a_{n_{\mu}}, b_{n_{\mu}}\right) \in \mathcal{g}_{r^{*}}, \mu=1,2, \cdots$, then, since $\bigcap\left\{\left(a_{m_{\nu}}, b_{m_{\nu}}\right): \nu=1,2, \cdots\right\} \cap\left(a_{n_{1}}, b_{n_{1}}\right) \neq \varnothing$, there is a $w \in X, w>a_{n_{1}}=r^{*}$, such that $w \in \cap\left\{\left(a_{m_{\nu}}, b_{m_{\nu}}\right): \nu=1,2, \cdots\right\}$. By construction of $\mathscr{g}_{0}$, $\cup\left\{\left(a_{m_{\nu}}, b_{m_{\nu}}\right): \nu=1,2, \ldots\right\}$ is contained in some member $I_{0}$ of $\mathfrak{g}_{0}$. Since $w \in I_{0}$ and $w>r^{*}$, it follows from the definition of $g_{0}$ that $I_{0}=(s, t]$. Moreover, since $\partial_{r^{*}}$ is an $\boldsymbol{\aleph}_{0}$-star refinement of itself, there is a $b>r^{*}$ such that $\left(r^{*}, b\right) \supset \bigcup\left\{\left(a_{n_{\mu}}, b_{n_{\mu}}\right): \mu=1,2, \cdots\right\}$. Let $c=\max \{t, b\}$. Then, by the choice of $r$, there is an interval $I \in \mathcal{g}$ such that $r \in I$ and $c \in I$. Since $r<s<r^{*}<c$, it follows that $\cup\left\{\left(a_{k}, b_{k}\right): k=1,2, \cdots\right\} \subset I$.

Similarly, if $\left(a_{m_{\nu}}, b_{m_{\nu}}\right) \in \mathcal{J}_{0}, \quad \nu=1,2, \cdots, \quad$ and $\left(a_{m_{\mu}}, b_{m_{\mu}}\right) \in J^{*}, \quad \mu$ $=1,2, \cdots$, then there is an $I \in g$ such that $\cup\left\{\left(a_{k}, b_{k}\right): k=1,2, \cdots\right\} \subset I$.

Since $l^{*}<r^{*}$, no member of $d l^{*}$ can meet a member of $g_{r^{*}}$. Therefore $\mathcal{g}$ is an open $\boldsymbol{\aleph}_{0}$-star refinement of $\mathscr{g}$, and hence of $R$. Hence $X$ is $\boldsymbol{\aleph}_{0}$-fully normal.

Case II. Suppose that both $u$ and $v$ are gaps, and that $X$ is not cofinal with $\omega$ but is coinitial with $\omega^{*}$. Define $r<s<r^{*}<t$ and $g_{r^{*}}$ as in Case I. Let $z_{1}, z_{2}, \cdots$ be an $\omega^{*}$-sequence coinitial with $X$ such that $z_{i}<t, i=1,2, \cdots$. Then $(u, t]=\bigcup\left\{\left[z_{i}, t\right]: i=1,2, \cdots\right\}$. Since $X$ has no interior gaps, it follows that each interval $\left[z_{i}, t\right]$ is compact. Therefore $(u, t]$ is $\sigma$-compact, i.e., the union of countably many compact subspaces. It is well-known that every regular $\sigma$-compact space is fully normal (cf. e.g. [10, Exercise 5.Y]). Therefore the linearly ordered space $(u, t]$ is fully normal.

Let $g_{0}=\left\{I \cap\left(u, r^{*}\right): I \in g\right\} \cup(\{(s, t]\}) . g_{0}$ is then an open (in $\left.(u, t]\right)$ covering of $(u, t]$ and hence admits an open (in $(u, t])$ star refinement $\mathcal{K}_{0}$ whose members are intervals of $(u, t]$. Let $\mathcal{J}_{0}=\left\{K-\{t\}: K \in \mathfrak{K}_{0}\right\}$ and $g=\partial_{0} \cup \partial_{r *}$. It follows, by an argument analogous to that employed in Case I, that $g$ is an open $\boldsymbol{\aleph}_{0}$-star refinement of $g$, and hence of $R$. Hence $X$ is $\boldsymbol{\aleph}_{0 \text { - }}$ fully normal.

Case III. Suppose that both $u$ and $v$ are gaps, and that $X$ is cofinal with $\omega$ but not coinitial with $\omega^{*}$. The argument in this case is analogous to that in Case II : the subspace $[p, v)$ is $\sigma$-compact, hence fully normal. 
Case IV. Suppose that $u$ is not a gap, $v$ is a gap, and $X$ is not cofinal with $\omega$. The proof is the same as in Case II: the subspace $[u, t]$ is compact, hence fully normal.

Case V. Suppose that $u$ is a gap, $v$ is not a gap, and $X$ is not coinitial with $\omega^{*}$. The proof is the same as in Case III: the subspace $[p, v]$ is compact.

Case VI. Suppose that none of the hypotheses of Cases I-V holds. Then $X$ is either compact or $\sigma$-compact, hence fully normal. This completes the proof of Lemma 3.5.

Corollary 3.6. If $X$ is a linearly ordered space, then the family of all neighborhoods of the diagonal in $X \times X$ is a uniformity for $X$ whose topology is the interval topology.

Proof. The assertion follows at once from Theorem 3.4 and Corollary 2.8.

4. Subspaces and products. J. Dieudonné [4, Théorème 2] has shown that each closed subspace of a paracompact space is itself paracompact, and that if each open subspace of a paracompact space is paracompact, then every subspace is paracompact. The first two theorems in this section establish the analogous results for $m$-fully normal and almost-m-fully normal spaces. The proofs which we give are similar to those used in [4].

Dieudonné $[4$, Théorème 5$]$ also proved that the cartesian product of a compact Hausdorff space and a paracompact space is paracompact. We give an example in this section to show that the analogous statement for $\boldsymbol{\aleph}_{0}$-fully normal spaces is false.

THEOREM 4.1. If $Y$ is a closed subspace of an $\mathfrak{m}$-fully normal (resp. almost$\mathfrak{m}$-fully normal) space $X$, then $Y$ is $\mathrm{m}$-fully normal (resp. almost-m-fully normal).

Proof. Let $R$ be a relatively open covering of $Y$. We must show that $R$ admits a relatively open $\mathfrak{m}$-star refinement (resp. almost-m-star refinement). There is a collection $R^{*}$ of open subsets of $X$ such that $R=\left\{R^{*} \cap Y: R^{*} \in R^{*}\right\}$. Let $\mathbb{R}^{* *}=\Omega^{*} \cup(\{X-Y\})$. Evidently $\mathfrak{R}^{* *}$ is an open covering of $X$. Therefore $R^{* *}$ admits an open $m$-star refinement (resp. almost-m-star refinement), say $\mathcal{S}^{*}$. Let $\mathcal{S}=\left\{S^{*} \cap Y: S^{*} \in \mathcal{S}^{*}\right\}$.

Evidently $\mathcal{S}$ is a relatively open covering of $Y$. We shall show that $\mathcal{S}$ is an $m$-star refinement (resp. almost-m-star refinement) of $R$.

Suppose that $J \subset S, \cap\{S: S \in J\} \neq \varnothing$, and $|\mathfrak{J}| \leqq \mathfrak{m}$ (resp. suppose that $M \subset$ St $(y, \delta)$ for some $y \in Y$ and $|M| \leqq m)$. For each $S \in J$ there is an $S_{S}^{*} \in \mathcal{S}^{*}$ such that $S=S_{S}^{*} \cap Y$. Now $\cap\left\{S_{S}^{*}: S \in J\right\} \neq \varnothing$ (resp. $M \subset$ St $\left(y, \delta^{*}\right)$ ). Since $S^{*}$ is an $m$-star refinement (resp. almost-m-star refinement) of $R^{* *}$, there is an $R^{* *} \in R^{* *}$ such that $U\left\{S_{S}^{*}: S \in J\right\} \subset R^{* *}$ (resp. such that $M \subset R^{* *}$ ). Since each $S_{S}^{*}$ contains points of $Y$ (resp. since $M \subset Y$ ), $R^{* *} \neq X-Y$. Hence $R^{* *}$ $\in R^{*}$. Hence $U\{S: S \in J\}=\bigcup\left\{S_{S}^{*}: S \in J\right\}=\bigcup\left\{S_{S}^{*}: S \in J\right\} \cap Y \subset R^{* *} \cap Y \in R$ (resp. $M \subset R^{* *} \cap Y \in R$ ). Therefore $\delta$ is an open m-star refinement (resp. almost-m-star refinement) of $(R$. 
An arbitrary subspace of an $\mathrm{m}$-fully normal space need not be almost-mfully normal: Let $X$ be a non-normal, completely regular, Hausdorff space $\left(^{6}\right)$. The Stone-Cech compactification $\left(^{7}\right) \beta X$ of $X$ is a fortiori $\mathfrak{m}$-fully normal for every $m \geqq 2$, but $X$ is, of course, not even normal.

THEOREM 4.2. If each open subspace of an m-fully normal (resp. almost-mfully normal) space $X$ is $\mathfrak{m}$-fully normal (resp. almost-m-fully normal), then every subspace of $X$ is $\mathfrak{m}$-fully normal (resp. almost-m-fully normal).

Proof. Suppose that $Y$ is a subspace of $X$, and let $R$ be a relatively open covering of $Y$. Then there is a collection $R^{*}$ of open subsets of $X$ such that $R=\left\{R^{*} \cap Y: R^{*} \in R^{*}\right\}$. Let $G=\cup\left\{R^{*}: R^{*} \in R^{*}\right\}$.

Now $G$, with the relative topology of $X$, is an open subspace of $X$, and $\Omega^{*}$ is a relatively open covering of $G$. Hence, by hypothesis, $\Omega^{*}$ admits an open (in $G$ and therefore in $X$ ) $\mathfrak{m}$-star refinement (resp. almost-m-star refinement) $\delta^{*}$. Let $\delta=\left\{S^{*} \cap Y: S^{*} \in \mathcal{S}^{*}\right\}$. It is easily seen that $\delta$ is an open (in $Y) \mathfrak{m}$-star refinement (resp. almost-m-star refinement) of $R$. Therefore $Y$ is $\mathfrak{m}$-fully normal (resp. almost-m-fully normal).

THEOREM 4.3. Let $(X, J)$ be an almost-2-fully normal Hausdorff space, and let $Y$ be a closed subset of $(X, \Im)$. Let $\mathfrak{J}_{Y}$ be the relativization of $\Im$ to $Y, \mathfrak{u}_{\Delta}$ the uniformity for $X$ consisting of all J-neighborhoods of the diagonal in $X \times X$, $\mathcal{U}_{\Delta_{Y}}$ the family of all $\Im_{Y}$-neighborhoods of the diagonal in $Y \times Y$, and $v_{Y}$ the relativization of $\mathfrak{u}_{\Delta}$ to $Y \times Y$. Then $\mathfrak{u}_{\Delta_{Y}}$ is a uniformity for $Y$ whose topology is $\jmath_{Y}$, and $u_{\Delta_{Y}}=\mathcal{V}_{Y}$.

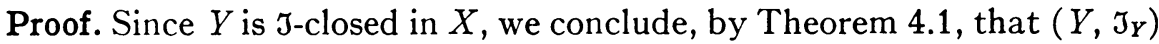
is almost-2-fully normal. Since $\left(Y, J_{Y}\right)$ is obviously a Hausdorff space, it follows from Corollary 2.8 that $\mathfrak{u}_{\Delta_{Y}}$ is a uniformity for $Y$ whose topology is $3_{Y}$

Now $\mho_{Y}$ is also a uniformity for $Y$ whose topology is $J_{Y}$ (cf. [10, p. 182]). Since $u_{\Delta_{Y}}$ is the largest (finest) uniformity for $Y$ with this property, we have $v_{Y} \subset \mathfrak{u}_{\Delta_{Y}}$. Therefore, in order to prove the last assertion of the theorem it suffices to show that $\mathfrak{u}_{\Delta_{Y}} \subset \mathcal{V}_{Y}$.

If $U \in \mathcal{u}_{\Delta_{Y}}$, then for each $y \in Y$ there is a $\Im_{Y}$-neighborhood $N_{y}$ of $y$ such that $N_{y} \times N_{y} \subset U$. For each $y \in Y$ there is a J-neighborhood $N_{y}^{*}$ of $y$ such that $N_{y}=N_{y}^{*} \cap Y$. Let $W^{*}=\left(\bigcup\left\{N_{\nu}^{*} \times N_{y}^{*}: y \in Y\right\}\right) \cup((X-Y) \times(X-Y))$. Since $Y$ is J-closed, it follows that $W^{*}$ is a J-neighborhood of the diagonal in $X \times X$, i.e. $W^{*} \in \mathcal{u}_{\Delta}$. Therefore $W=W^{*} \cap(Y \times Y) \in \mathcal{V}_{Y}$.

If $(p, q) \in W$, then $(p, q) \in W^{*}$ and $p, q \in Y$. Hence $(p, q) \notin(X-Y)$ $\times(X-Y)$. Therefore, by definition of $W^{*}$, there is a $y \in Y$ such that $(p, q)$ $\in N_{y}^{*} \times N_{\nu}^{*}$. Hence $(p, q) \in\left(N_{\nu}^{*} \cap Y\right) \times\left(N_{\nu}^{*} \cap Y\right)=N_{y} \times N_{y} \subset U$. We conclude that $W \subset U$, and therefore $U \in \mathcal{V}_{Y}$. Consequently $\mathfrak{u}_{\Delta_{Y}} \subset \mathcal{V}_{Y}$.

The cartesian product of two m-fully normal spaces need not be normal: In his paper [14], R. H. Sorgenfrey gives an example of a space $S$ (the set of

${ }^{(6)}$ See, for example, [10, Exercise 4.E].

(7) See, for example, $[10$, p. 152$]$. 
non-negative real numbers with the "half-open interval" topology) with the property that $S$ is paracompact-and hence $\mathfrak{m}$-fully normal for any $\mathfrak{m}$-but $S \times S$ is not normal.

The cartesian product of an $\boldsymbol{\aleph}_{0}$-fully normal space and a compact space need not be normal: The space $W\left(\omega_{1}\right) \times W\left(\omega_{1}+1\right)$ is not normal (cf. e.g. [10, Exercise 4.E]). As we shall see later, the cartesian product of an almost- $\boldsymbol{\aleph}_{0^{-}}$ fully normal space and a compact metrizable space is normal.

5. Properties. In this section we derive several properties of $m$-fully normal and almost-m-fully normal spaces. Most of these properties have analogs in the theory of paracompact spaces.

Definition 5.1. Let $m$ be an infinite cardinal number. A collection $a$ of subsets of a topological space $X$ is said to be locally-m if each $x \in X$ has a neighborhood meeting at most $\mathfrak{m}$ members of $a$.

Our first theorem is a generalization of a result due to J. L. Kelley [10, Lemma 5.31].

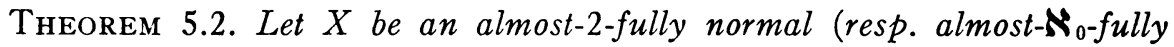
normal, resp. almost- $\boldsymbol{\aleph}_{\alpha+1}$-fully normal) space. If $a$ is a discrete (resp. locally finite, resp. locally $-\boldsymbol{\aleph}_{\alpha}$ ) collection of subsets of $X$, then there is an open symmetric neighborhood $V$ of the diagonal in $X \times X$ such that the family $\{V[A]$ : $A \in Q\}$ is discrete (resp. locally finite, resp. locally-న $\boldsymbol{\aleph}_{\alpha}$ ).

Proof. Let $Q$ be a discrete (resp. locally finite, resp. locally- $\boldsymbol{\aleph}_{\alpha}$ ) collection of subsets of $X$. Then there is an open covering $R$ of $X$ such that each member of $R$ meets at most one member (resp. only finitely many members, resp. at

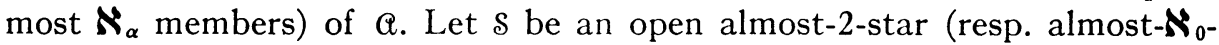
star, resp. almost- $\aleph_{\alpha+1}$-star) refinement of $R$, and set $U=U\{S \times S: S \in \mathcal{S}\}$. Since $X$ is almost-2-fully normal, there is an open symmetric neighborhood $V$ of the diagonal in $X \times X$ such that $V \circ V \subset U$ (cf. Theorem 2.6).

We shall show that $\{V[A]: A \in a\}$ is a discrete (resp. locally finite, resp. locally-秋) collection. If $V[x] \cap V[A] \neq \varnothing$ for some $x \in X$ and some $A \in Q$, choose $z \in V[x] \cap V[A]$. Then there is a $p_{A} \in A$ such that $\left(p_{A}, z\right) \in V$. Since $(x, z) \in V$ and $V$ is symmetric, we conclude that $\left(x, p_{A}\right) \in V \circ V \subset U$. Hence, by definition of $U$, there is an $S_{x, A} \in \delta$ such that $x \in S_{x, A}$ and $p_{A} \in S_{x, A}$.

Suppose that there is an $x \in X$ such that $V[x]$ meets more than one member (resp. infinitely many members, resp. more than $\boldsymbol{\aleph}_{\alpha}$ members) of $\{V[A]$ : $A \in Q\}$. Then there is a $B \subset Q$ such that $|B|=2$ (resp. $|B|=\boldsymbol{N}_{0}$, resp. $|B|$ $=\boldsymbol{\aleph}_{\alpha+1}$ ), and such that $B \in B$ implies that $V[x] \cap V[B] \neq \varnothing$. Hence, by the preceding paragraph, for each $B \in \Theta$ there is a $p_{B} \in B$ and a set $S_{x, B} \in \mathcal{S}$ such that $x \in S_{x, B}$ and $p_{B} \in S_{x, B}$. Hence $\left\{p_{B}: B \in \mathbb{B}\right\} \subset \operatorname{St}(x, \delta)$. Since $\mathcal{S}$ is an

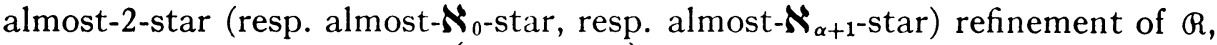
there is an $R \in R$ such that $\left\{p_{B}: B \in \Omega\right\} \subset R$. This contradicts the definition of $R$. Hence, for each $x \in X$, the open set $V[x]$ meets at most one member (resp. only finitely many members, resp. at most $\boldsymbol{\aleph}_{\alpha}$ members) of $\{V[A]$ : $A \in Q\}$. 
Corollary 5.3. If $X$ is an almost- $\boldsymbol{\aleph}_{0}$-fully normal space, then $X$ is countably paracompact $\left.{ }^{8}\right)$.

Proof. It is shown in [11] that a normal space $X$ is countably paracompact if (and only if) for each countable locally finite collection $\left\{A_{i}: i=1,2, \cdots\right\}$ of subsets of $X$ there is a countable, locally finite collection $\left\{G_{i}: i=1,2, \cdots\right\}$ of open subsets of $X$ such that $A_{i} \subset G_{i}, i=1,2, \cdots$.

The converse of Corollary 5.3 is false. R. H. Bing [1, Example H] has given an example of a perfectly normal space $F$ which is not collectionwise normal. Since $F$ is perfectly normal, $F$ is countably paracompact (cf. [6, Corollary to Theorem 2]). On the other hand, $F$ cannot be almost-2-fully normal, since $F$ is not collectionwise normal (cf. Theorem 2.9).

The example due to Dowker following Definition 2.3 shows that we cannot replace "almost- $\boldsymbol{\aleph}_{0}$-fully normal" by "finitely-fully normal" in the hypothesis of Corollary 5.3. As remarked before, Dowker's space $X$ is finitely fully normal. The space $X$ is not countably paracompact because the countable open covering $\left\{G_{i}: i=1,2, \cdots\right\}$, where $G_{i}=\{x \in X: x<i\}$, does not admit a locally finite open refinement. It is an open question, of course, as to whether every finitely-fully normal Hausdorff space is countably paracompact.

Observe that Theorem 5.2 contains Theorem 2.9.

It has been shown by Dowker [6, Lemma 3] that the cartesian product of a countably paracompact normal space and a compact metrizable space is normal. Hence it follows from Corollary 5.3 that the cartesian product of an almost $\boldsymbol{\aleph}_{0}$-fully normal space and a compact metrizable space is normal.

It follows from Corollary 5.3 that Theorem 3.4 is a stronger result than [7, Theorem 9.5], which states, in part, that every linearly ordered space is countably paracompact.

Definition 5.4. Let $m$ be any cardinal number $\geqq 2$. A covering $R$ of a topological space $X$ is said to be $m$-even if there is a neighborhood $U$ of the diagonal in $X \times X$ such that if $M \subset U[x]$ for some $x \in X$ and $|M| \leqq \mathfrak{m}$, then there is an $R \in R$ such that $M \subset R$.

Our next result is similar to [10, Theorem $5.28(\mathrm{~d})]$. which says that a regular Hausdorff space is paracompact if and only if each open covering is evell.

Theorem 5.5. A topological space $X$ is almost-m-fully normal if and only if each open covering of $X$ is m-even.

Proof. Suppose that each open covering of $X$ is m-even. Let $R$ be an open covering of $X$; we shall show that $R$ admits an open almost-m-star refinement. By hypothesis, there is a neighborhood $U$ of the diagonal in $X \times X$ such that $M \subset U[x]$ for some $x \in X$ and $|M| \leqq \mathrm{m}$ imply that there is an $R \in R$ with

(8) See footnote 3 . 
the property that $M \subset R$.

For each $x \in X$ there is an open set $W_{x}$ containing $x$ such that $W_{x} \times W_{x} \subset U$. The family $\left\{W_{x}: x \in X\right\}$ is then an open covering of $X$. Therefore there is a neighborhood $V$ of the diagonal in $X \times X$ such that if $\{p, q\} \subset V[x]$ for some $x \in X$, then there is a $z \in X$ such that $\{p, q\} \subset W_{z}$. Let $V^{\prime}=\operatorname{Int} V$ and $V^{\prime \prime}$ $=V^{\prime} \cap V^{\prime-1}$. Now if $(u, v) \in V^{\prime \prime} \circ V^{\prime \prime}$, then there is a $w \in X$ such that $(u, w) \in V^{\prime \prime}$ and $(w, v) \in V^{\prime \prime}$. Since $V^{\prime \prime}$ is symmetric, this means that $u, v \in V^{\prime \prime}[w] \subset V^{\prime}[w] \subset V[w]$. Hence, by the choice of $V$, there is an $x \in X$ such that $\{u, v\} \subset W_{x}$. Hence $(u, v) \in W_{x} \times W_{x} \subset U$. Therefore $V^{\prime \prime}$ is an open symmetric neighborhood of the diagonal such that $V^{\prime \prime} \circ V^{\prime \prime} \subset U$.

For each $x \in X$ choose $R_{x} \in R$ such that $x \in R_{x}$, and let $S_{x}=V^{\prime \prime}[x] \cap R_{x}$. Then $\mathcal{S}=\left\{S_{x}: x \in X\right\}$ is an open refinement of or. Suppose that $y \in \operatorname{St}(x, \mathcal{S})$ for some $x \in X$. Then there is a $z \in X$ such that $x, y \in S_{z} \subset V^{\prime \prime}[z]$. Since $V^{\prime \prime}$ is symmetric, this means that $(x, y) \in V^{\prime \prime} \circ V^{\prime \prime} \subset U$. Thus $y \in U[x]$. Hence St $(x, S) \subset U[x]$. It follows that $S$ is an open almost-m-star refinement of $R$.

Conversely, suppose that $X$ is almost-m-fully normal. Let $R$ be an open covering of $X$. We must show that $R$ is $\mathrm{m}$-even. By hypothesis, $R$ admits an open almost-int-star refinement $\delta$. Let $U=U\{S \times S: S \in \delta\}$. Evidently $U$ is a neighborhood of the diagonal in $X \times X$.

Suppose $M \subset U[x]$ for some $x \in X$ and $|M| \leqq m$. Then, by definition of $l$, for each $y \in M$ there is a set $S_{u} \in \mathcal{S}$ such that $x, y \in S_{y}$. Therefore

$$
M \subset \mathrm{St}(x, \mathrm{~s}),
$$

and so there is an $R \in R$ such that $M \subset R$. Hence $(R$ is m-even.

Definition 5.6. (a) A net $\left.{ }^{9}\right)\left\{S_{n}, n \in D\right.$, $\left.\geqq\right\}$ is called an $\mathfrak{m}-n e t$ ( $m$ any infinite cardinal number) if for each $n \in D$ the set $D_{n}=\{p \in D: p \geqq n\}$ has power at most $\mathrm{m}$.

(b) A uniform space $(X, \mathcal{u})$ is said to be $\mathrm{nt}$-complete if each Cauchy m-net in $(X, \mathcal{u})$ converges in $X$ relative to the uniform topology.

J. Nagata [13] and Alice Dickinson [3] have shown (independently) that if $X$ is a paracompact space and $u_{\Delta}$ is the family of all neighborhoods of the diagonal in $X \times X$, then $\left(X, \mathfrak{u}_{\Delta}\right)$ is a complete uniform space $\left({ }^{10}\right)$. Our next theorem describes the situation for almost-m-fully normal spaces.

Theorem 5.7. If $(X, \mathfrak{J})$ is an almost-m-fully normal Hausdorff space for some infinite cardinal number $\mathfrak{m}$, and if $\mathfrak{u}_{\Delta}$ denotes the uniformity for $X$ consisting of all J-neighborhoods of the diagonal in $X \times X$, then the uniform space $\left(X, \mathfrak{u}_{\Delta}\right)$ is $\mathrm{m}$-complete.

Proof. We first observe that, in virtue of Corollary 2.8, the uniform topology and $J$ are identical. Suppose that $\left\{S_{n}, n \in D, \geqq\right\}$ is a Cauchy $\mathrm{m}$-net in $\left(X, \mathcal{u}_{\lrcorner}\right)$which does not converge in $X$. Then $\left\{S_{n}, n \in D, \geqq\right\}$ has no cluster points in $X$. Therefore for each $x \in X$ there is an open neighborhood $G_{x}$ of $x$

(9) See [10, Chap. 2].

(10) See [10, Chap. 6]. 
and an $n_{x} \in D$ such that $n \geqq n_{x}$ implies that $S_{n} \in X-G_{x}$. The family $\left\{G_{z}: z \in X\right\}$ is then an open covering of $X$. Since $X$ is almost-m-fully normal, there is, according to Theorem 5.5, a $U \in \mathcal{u}_{\Delta}$ such that if $M \subset U[x]$ for some $x$ and $|M| \leqq \mathfrak{m}$, then there is a $z \in X$ such that $M \subset G_{z}$.

Now, since $\left\{S_{n}, n \in D, \geqq\right\}$ is a Cauchy net, there is an $n_{U} \in D$ such that $p \geqq n_{U}$ implies that $\left(S_{n_{U}}, S_{p}\right) \in U$. Hence if $p \geqq n_{U}$, then $S_{p} \in U\left[S_{n_{U}}\right]$. Since the set $\left\{p \in D: p \geqq n_{U}\right\}$ has, by hypothesis, power at most $\mathfrak{m}$, it follows from the choice of $U$ that there is a $z \in X$ such that $\left\{S_{p}: p \geqq n_{U}\right\} \subset G_{z}$. Choose $p_{0} \in D$ such that $p_{0} \geqq n_{U}$ and $p_{0} \geqq n_{z}$. Then $S_{p_{0}} \in G_{z}$, and, by choice of $n_{z}$, $S_{p_{0}} \in X-G_{z}$. This is impossible. Therefore each Cauchy m-net in $\left(X, \mathfrak{u}_{\Delta}\right)$ converges in $X$. Consequently $\left(X, \mathfrak{u}_{\Delta}\right)$ is $\mathfrak{m}$-complete.

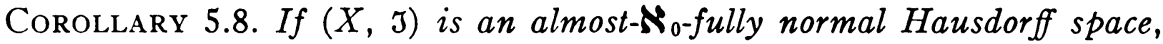
then each Cauchy sequence in $\left(X, \mathfrak{u}_{\Delta}\right)$ converges in $X$.

Proof. A Cauchy sequence is evidently a Cauchy $\boldsymbol{\aleph}_{0}$-net.

A covering $R$ of a set $X$ is said to be point-finite if each $x \in X$ is contained in only a finite number of members of $R$.

Definition 5.9. Let $m$ be an infinite cardinal number. A covering $R$ of a set $X$ is said to be point- $\mathfrak{m}$ if each $x \in X$ is an element of at most $\mathfrak{m}$ members of $R$.

E. Michael [12, Theorem 2] has shown that if $X$ is a collectionwise normal Hausdorff space such that each open covering of $X$ admits an open pointfinite refinement, then $X$ is paracompact. Our next result is of the same nature as Michael's theorem.

THEOREM 5.10. Let $\mathrm{m}$ be an infinite cardinal number. If a topological space $X$ is $\mathrm{m}$-fully normal, and if each open covering of $X$ admits an open point- $\mathfrak{n}$ refinement for some $\mathfrak{n} \leqq \mathfrak{m}$, then $X$ is fully normal.

Proof. Let $R$ be an open covering of $X$. We must show that $R$ admits an open star refinement $\mathfrak{J}$. By hypothesis, $\mathcal{R}$ admits an open $m$-star refinement $\mathcal{S}$, and $\mathcal{S}$ admits an open point- $\mathfrak{n}$ refinement $\mathfrak{J}$ for some $\mathfrak{n} \leqq \mathfrak{m}$.

If $x \in X$, let $\mathfrak{J}_{x}=\{T \in \mathfrak{J}: x \in T\}$. Since $\mathfrak{J}$ is point- $\mathfrak{n}$, we have $\left|J_{x}\right| \leqq \mathfrak{n} \leqq \mathfrak{m}$. For each $T \in J_{x}$ choose $S_{T} \in \mathcal{S}$ such that $T \subset S_{T}$. Since $x \in \cap\left\{S_{T}: T \in J_{x}\right\}$ and $S$ is an m-star refinement of $R$, there is an $R \in R$ such that $U\left\{S_{T}: T \in J_{x}\right\} \subset R$. Consequently St $(x, \mathfrak{J})=\bigcup\left\{T: T \in J_{x}\right\} \subset \cup\left\{S_{T}: T \in J_{x}\right\} \subset R$. Therefore $\mathfrak{J}$ is an open star refinement of $R$. Hence $X$ is fully normal.

Theorem 5.11. Let $X$ be an $\boldsymbol{\aleph}_{\beta+1}$-fully normal space. If $R=\left\{R_{\alpha}: \alpha \in A\right\}$ is an open covering of $X$ such that $|A|=\boldsymbol{\aleph}_{\beta+1}$, then there is an open point $-\boldsymbol{\aleph}_{\beta}$ covering $S=\left\{S_{\alpha}: \alpha \in A\right\}$ of $X$ such that $S_{\alpha} \subset R_{\alpha}$ for each $\alpha \in A$.

Proof. Let $R=\left\{R_{\alpha}: \alpha \in A\right\}$ be an open covering of $X$ such that $|A|$ $=\boldsymbol{\aleph}_{\beta+1}$. We may assume, without loss of generality, that $A=W\left(\omega_{\beta+1}\right)$. Let $\Im$ be an open $\boldsymbol{\aleph}_{\beta+1}$-star refinement of $R$.

For each $\alpha \in A$ put $S_{\alpha}=\bigcup\left\{T \in J: T \subset R_{\alpha}\right.$ and if $\gamma<\alpha$, then $\left.T \llbracket R_{\gamma}\right\}$. 
Clearly $S_{\alpha} \subset R_{\alpha}$ for each $\alpha \in A$. If $x \in X$, then $x \in T$ for some $T \in$ J. Let $\alpha_{x}$ be the smallest ordinal $\alpha \in W\left(\omega_{\beta+1}\right)$ such that $T \subset R_{\alpha}$. It follows that $x \in S_{\alpha_{x}}$. Hence $S=\left\{S_{\alpha}: \alpha \in A\right\}$ is an open covering of $X$.

Suppose that there is an $x_{0} \in X$ such that $x_{0}$ is an element of more than $\boldsymbol{\aleph}_{\beta}$ members of $\delta$, say $x_{0} \in S_{\alpha_{\delta}}, \delta \in D$, where $|D|=\boldsymbol{\aleph}_{\beta+1}$. By definition of $\mathcal{S}$, for each $\delta \in D$ there is a $T_{\delta} \in \mathcal{J}$ such that $x_{0} \in T_{\delta} \subset S_{\alpha_{\delta}}$. Hence $\cap\left\{T_{\delta}: \delta \in D\right\}$ $\neq \varnothing$, and so, since $J$ is an $\boldsymbol{\aleph}_{\beta+1}$-star refinement of $R$, there is an $\alpha^{*} \in W\left(\omega_{\beta+1}\right)$ such that $\cup\left\{T_{\delta}: \delta \in D\right\} \subset R_{\alpha^{*}}$.

Now $T_{\delta} \subset S_{\alpha_{\delta}}$ means, by definition of $S_{\alpha_{\delta}}$, that $T_{\delta} \subset R_{\alpha_{\delta}}$ and if $\gamma<\alpha_{\delta}$, then $T_{\delta} \subset R_{\gamma}$. But $T_{\delta} \subset R_{\alpha^{*}}$ for every $\delta \in D$, and so $\alpha^{*} \geqq \alpha_{\delta}$ for every $\delta \in D$. This is impossible because $\omega_{\beta+1}$ is the smallest ordinal with $\boldsymbol{\aleph}_{\beta+1}$ predecessors, and $\omega_{\beta+1}>\alpha^{*}$. Hence each point $x \in X$ is an element of at most $\boldsymbol{\aleph}_{\beta}$ members of $\delta$. Therefore $S$ is an open point $-\boldsymbol{\aleph}_{\beta}$ refinement of $R$.

\section{REFERENCES}

1. R. H. Bing, Metrization of topological spaces, Canadian Journal of Mathematics vol. 3 (1951) pp. 175-186.

2. H. J. Cohen, Sur un problème de M. Dieudonnê, C.R. Acad. Sci. Paris vol. 234 (1952) pp. 290-292.

3. Alice Dickinson, Compactness conditions and uniform structures, Amer. J. Math. vol. 75 (1953) pp. 224-227.

4. J. Dieudonné, Une généralisation des espaces compacts, J. Math. Pures Appl. vol. 23 (1944) pp. 65-76.

5. - Un exemple d'espace normal non susceptible d'une structure uniforme d'espace complet, C.R. Acad. Sci. Paris vol. 209 (1939) pp. 145-147.

6. C. H. Dowker, On countably paracompact spaces, Canadian Journal of Mathematics vol. 3 (1951) pp. 219-224.

7. L. Gillman and M. Henriksen, Concerning rings of continuous functions, Trans. Amer. Math. Soc. vol. 77 (1954) pp. 340-362.

8. A. J. Goldman, $A$ Cech fundamental group, Summary of lectures and seminars of the Summer Institute on Set Theoretic Topology, Madison, Wisconsin, American Mathematical Society Summer Institute, 1955, pp. 107-109.

9. F. Hausdorff, Grundzïge der Mengenlehre, Leipzig, Viet, 1914. (Reprinted New York, Chelsea, 1949.)

10. J. L. Kelley, General topology, New York, van Nostrand, 1955.

11. M. J. Mansfield, On countably paracompact normal spaces, Canadian Journal of Mathematics vol. 9 (1957) pp. 443-449.

12. E. Michael, Point-finite and locally finite coverings, Canadian Journal of Mathematics vol. 7 (1955) pp. 275-279.

13. J. Nagata, On topological completeness, J. Math. Soc. Japan vol. 2 (1950) pp. 44-47.

14. R. H. Sorgenfrey, On the topological product of paracompact spaces, Bull. Amer. Math. Soc. vol. 53 (1947) pp. 631-632.

15. A. H. Stone, Paracompactness and product spaces, Bull. Amer. Math. Soc. vol. 54 (1948) pp. 977-982.

16. J. W. Tukey, Convergence and uniformity in topology, Princeton, Princeton University Press, 1940.

Purdue University, LAFAYETTE, IND. 